\title{
Neuro-psycho-education-existential group program: Evidence based Professional Practice at persons with psychiatric and intellectual disabilities.
}

\author{
Suela Ndoja ${ }^{1}, M S c$, PhD Cand.
}

\begin{abstract}
:
Aim: to describe the impact of neuro-psycho-education-existential group program on personal formation and its possible application for mental health, health and social professions in different contexts by taking into consideration the current practice with psychiatric and intellectual disability persons.

Framework: the theoretical foundations of clinically based in combination of perspectives as neuropsychology, psycho education and existentialism are reviewed and the common positive elements of practice are identified.

Practical application: examples of well-defined ten weekly neuro-psycho-educationexistential interventions in group developed in public spaces are presented that meet criteria for empirically supported psychological interventions at persons with psychiatric and intellectual disabilities.

Outcomes: in conclusion, the specific application of neuro-psycho-education-existential group program for health care, mental health and social practice at both the clinical and community disability level is discussed.

Implications: the need for further evaluation and design research is considered for a better implementation into practice.
\end{abstract}

Keyword: neuro-psycho-education-existential group program, persons with psychiatric and intellectual disabilities, Evidence based Professional Practice.

\section{Introduction}

The aim of this project pilot is to describe the impact of neuro-psycho-educationexistential group program (NPEEGP) on personal formation and its possible application for mental health, health and social professions in different contexts by taking into consideration the current practice with psychiatric and intellectual disability persons. (PIDP)

This project pilot specifically targets younger and adults with a psychiatric and intellectual disability.There are included 28 persons with psychiatric and

${ }^{1}$ Clinical Psychologist, Italian-Albanian Association, "Progetto Speranza" 
intellectual disability aged 16-35 years old including 10 adults ( 8 boys and 2 girls) with a psychiatric disability under the effects of medication and 18 younger and adults (10 boys and 8 girls) with intellectual disability who have a diagnosis of mental retardation and cognitive deficits (those who require assessment because their behavior suggests they may have a dual diagnosis). They all live in Homes-Family and are social or biological orphans who are grown up in Orphaned Institutions and Psychiatric Centers.

\subsection{Background}

The impact of PPID experience has long been a historical, theoretical and clinical interest; it is not something new. PPID are at increased risk of mental health problems when compared to the general population. The differing presentations and associated needs is very confusing to some professionals especially mentioning here clinical psychologists and consequently means there is no standard response when working with a young or an adult who has an psychiatric or an intellectual disability and co-existing mental health problems or other secondary pathologies. General practitioners, mental health and disability social service providers feel that only when problems get to crisis point is there some chance of a service or intervention response. They are not never trained enough to identify and analyze how psychopathology is masked or distorted by the presence of a psychiatric and intellectual disability. That's why Ivanov Smolenski writes: "Non e' facile stabilire fin dove arriva il fisiologico e dove incominca il patologico,quanto entrano in arione i meccanismi di difesa: nella misura in cui impediscono la normale attivita sono patologici, nella misura in cui proteggono la cellula sono normali" Marzuoli U,1961: 230-231). The complex needs of these persons translate into difficulties in supporting and sustaining them especially when they are also orphans. They experience significant challenges in regard to meeting their clinical needs within this context based on accommodation options. Despite residential options, strong plan care that suit the needs of these persons, they often appear to fall into the "too hard basket" because there are no clear solutions or responses to problems and needs being experienced.

\subsection{General issues on persons with psychiatric and intellectual disabilities-}

Resource constraints and planning problems have resulted in groups of younger and adults with a psychiatric and an intellectual disability living together who are incompatible. Thus, behavioral disorders always accompany their living which clearly constitutes a significant clinical and vital concern. When the appearance of these disorders is not treated, they can develop serious, threatening actions 
towards one self and each-other. It is important to stress that concerns relate to the provision of the "right mix" of interventions that are available to them. For example, when these persons present with challenging behavior, assessment needs to rule mental health problems either "in" or "out" and then try to educate them how to live with one's incapacity in order to give a meaning to one's existence but this one can't happen immediately or without a stimuli. The nature of problems is not only in PPID itself but also to the general practitioners, mental health and disability service providers. Too many psychological studies are conducted on PPID, however there are no specific studies conducted on the education of their existence including environment. Therefore is obvious that it comes to a great and valuable investment; trying always by adding new programs of interventions who maybe as a complement in plan care.

\section{Current clinical practice with psychiatric and intellectual disabilities:}

It was February 2011, when I began to work at Progetto Speranza for persons with disabilities. Driven by my interest for these persons, I began passionately working in Diurnal Center of Occupational Therapy and Socialization trying to apply Neuro-psycho-education Model (Ndoja S, 2012: 68) to which it will be given a considerable description later. After one year knowing better the problems and the special needs of these persons, especially the need to be stimulated, to be psychologically supported and to be educated regarding their existence; it seemed necessary to move forward, which made me a very engaged clinical operator.Benefits of being a part of the multidisciplinary team gave me the opportunity to experience how the other disciplines saw their development. It was the time when I began formulating an understanding of complex painting to every one of them with disability. For egg working with educators, illustrated the impact of everyday life in socio-emotional aspects and atypical behaviors during the staying at home-family. Also from psychiatrics, the illustration of behavior problems or mental health regarding side effects of drug therapy. All this presented to me a considerable challenge. Doing further, it became clear to me that it was not the question of elaborating a program of intervention, but also to develop and integrate more under questions including understanding how various issues could affect or dominate each other during its application. I realized that contributions of discipline's psychology were only part of the complex picture that builds the range of work with PPID because basically the picture involves understanding how the current behavior is influenced by many factors including among others the environment. It should be noted significantly that application of this program has benefits and disadvantages too. So for practitioners who are interested in working with PPID, primary focus should be on the jamming that are buzzers of special needs characteristics, typical and 
atypical behavior and mental health problems. Since the world of disabilities is a time of rapid change (progress and regress) based on my experience, should be included best practices assessment models, and to consult for a better functioning of their existence. It's impliedly that no intervention program or method is suitable for all. However, it can be encouraged the use of typical group program as may be set in this case the neuro-psycho-educative-existential group program. Let's see below what I mean by this...

\section{Theoretical Framework: clinical intervention description}

Given my two years experience at Progetto Speranza and taking into consideration the aspiration to elaborate interventions based on their vital needs, it seemed necessary to design a group program which reflect on learning of being stimulated, feeling psychologically supported, doing, living together and existing not simply being, because in this way the professions' create and maintain a humane system of education for the existence of these persons. Group program is based on a review of theoretical foundations combination of three perspectives, neuropsychology, psycho education and existentialism.

\subsection{Review of theoretical foundations of presuppositions in neuropsychology}

Here neuropsychology, as effect of intervention refers to the mind, the way how does a person think, refers to the nervous system and how the disfuncioned brain elaborates the guides. (Ndoja S, 2012: 68-69). It is divided in:

a. Operational system of presupposition based on presupposed beliefs- which are a basic structure in model of the world that is seen as a reality. Here the beliefs are called basic beliefs and they act as filters that determine how we perceive external reality.

b. Presuppositions of mental processing based on Representative system- in which are set all experiences as a result of what we hear, see, feel, touch and smell, for this through the five senses. Although that human beings share the five senses and their neurology basis, their unique experience changes as our five fingers. And this is a big difference between persons.

c. Presuppositions of mental processing based on Maps and Filters in which when a person describes his/her world, he/she uses it on two levels: an objective, external reality and internal reality subjective. At this group program the "map is not the territory." Territory is the reality and the map describes what 
exists in the mind of the person's mental understanding of the territory. The group program wants to change the map but not the reality.

5.2. Review of theoretical foundations of presuppositions in psychoeducation

Here psycho-education is based on the work of psychologists as Fritz Pearls, Virginia Satire and Milton Erikson:

$i$. Fritz Perls- there are taken into consideration techniques of Pearls as following: Here and now, Contact, Learning through experience and Internal dialogues.

ii. Virginia Satir- there are taken into consideration techniques of Satir as following: Erasures-Reframing, Anchoring and Gentleness.

iii. Milton Ericson- there are taken into consideration techniques of Satir: Sources, Report, Rhythm; moving the person toward the desire to give the relevant opinion

$i v$. Robert Dilts - created a mode; of personal change, according which the person can be educated in six levels: spiritual, identity, beliefs and values, skill, behavior and environment. (Dilts R.1996)

\subsection{Review of theoretical foundations of presuppositions in existentialism}

Here existentialism is based on the principles of deep questions about the nature of the human being and the nature of anxiety, despair, grief, loneliness, isolation, and anomie. It also deals centrally with the questions of creativity and love. It may be reflected upon how the client has answered life's questions in the past, but attention ultimately shifts to searching for a new and increased awareness in the present and enabling a new freedom and responsibility to act. The person can then accept they are not special, and that their existence is simply coincidental by accepting concepts of "I-Am" Experience and the Significance of Time

\subsection{Central Techniques of Neuro-Psycho-educative-existential Group Program}

Management of mental status - Changing the situation changes all the time and this can be done by anchoring that may refer to a stimulus to change a position to recreate a condition. Emotional mood is a way of being in any given moment. (McDermott \& Jago 2001). 
- $\quad$ Setting goals -Present state and desired state - the model wants to look to the desired state more than the state of present problems. Abraham Maslow states that a goal gives purpose and direction and is the first step towards his/her achievement.

\subsection{What should be taken into account in neuro-psycho-education- existential group program?}

General practitioners, mental health and disability social service providers should:

First: be qualified and implement the Professional Ethic.

Secondly: get necessary information which includes characteristics and beliefs of PPID, psychological, health, neurodevelopment, behavioral issues, developmental levels in all areas, relationships with others, community stressors and environment resources.

Thirdly: learn to evaluate the ability of PPID by a formal assessment tool, not testing, the PPID's ability to ignore distraction in the environment and interest. Such factors can provide a more comprehensive picture of their functioning and can provide a greater insight to intervention plan.

Fourthly: build a cooperative relationship as a context for personal and professional development and conduct detective conversations with caregivers of PPID regarding their mental states and psychological mood.

Fifthly: have the ability to develop programs according the problems and clinical needs of PPID.

Sixthly: create a warm and comprehensive atmosphere in suitable environments in which it is prepared every necessary tool as materials etc.

Seventhly: be able adapting to unusual situations that may occur during the intervention group program. Here the program must currently be in second place. Furthermore there should be a clinical fit between the clinician, the persons with disability personality and individual comfort zone in this area.

Lastly: understand how group program will be used for the issuance of recommendations and treatment plans. Interpreting of complex data and writing a clearly report does not happen very soon.

\section{Evidence based Professional Practice: Description of Practical application}

Ethical approval was granted by Professional Staff of Proggetto Speranza. Informed consent for participation was received from each participant by 5 May 2012 , in the first day. 
Participants- Individuals who satisfied selection criteria (Psychiatric and Intellectual disability) were informed of the group program developed in public spaces, and invited to participate. The participant wishing to be involved in the study was informed of the aims and purpose of the research, the nature of their involvement, how the data will be used and their right to withdraw from the study at any time.

Assistants of group program: there have participated three persons (one girl and two boys) with intellectual disabilities from Home Families who have been included in preparation and organization of program. Also every Sunday is invited an educator by the PPID themselves.

Intervention- The key feature of this project pilot was the design and implementation of a NPEEGP specifically for PPID. The intervention was a ten weekly program, focusing on (a) giving a stimulus as outside activity in public spaces, (b) building psychological and social support to make them feel better (c) introducing skill-building exercises, (d) educating about personal living inside and outside the basket regarding existence and (e) physic and mental exercises. The contents of these sessions are detailed in Table below. The twenty - eight participants assigned to the intervention group participated in weekly three-hour social over a 10 weekly period (May- June 2012) with an interruption on July and then the two last sessions were implemented along September 2012 period. It is called the Road of Hope Activity. This intervention was both individualized and group based with a focus on participants' effective management of their existence, associated participation in community social and recreational activities.

Table 1: Content of ten weekly neuro-psycho-education-existential group programs

\begin{tabular}{|l|l|l|}
\hline & Sessions & Goals \\
\hline 1 & Opening : & Mission and Vision \\
\hline 2 & Identity \& Existence: & Who I am \& do I exist? \\
\hline 3 & Spiritual: & What is my purpose; do I have a meaning in my life? \\
\hline 4 & Values and beliefs: & Why I do certain things? \\
\hline 5 & Skills: & How I am doing and actually do certain things: fear? \\
\hline 6 & Behavior: & $\begin{array}{l}\text { What I am doing, how do I behave with myself and with } \\
\text { others? }\end{array}$ \\
\hline 7 & Orientation: & Where I have been and do I am right now? \\
\hline 8 & Capabilities: & How can I solve my problems and meet my needs? \\
\hline 9 & Environment: & Where I am going to be in my future and when? \\
\hline 10 & Closure & Reflections, cocktail and certifications of participation \\
\hline
\end{tabular}




\subsection{A summary of neuro-psycho-educative-existential methods is detailed below.}

a. Stimulus -Sessions included giving the first stimulus as going and teaching "out strong basket" by being activated following a regular line as the place of meeting in the morning, the choice of location, departure, arrival in the location at public spaces, the establishment of large group, hospitality of educators invited by themselves through votes, sharing in mini-groups, continuation of the first part of the program, the pause of 15 minutes, continuation of the second part of the program, the pursuit of physical and mental exercises, phase of reflection, sharing of individual homework, the serving as ice-cream ecc, the way to homefamily.

b Psychological and Social Support- Participants were involved in an ongoing psychological and social support program to assist in their development. Activities were recreationally and socially oriented (e.g., mini-group preparatory work including persons with whom they didn't have good remarks), with benefits including feedback and social support from others, as well as learning more values, selfregulation, motivation and awareness. In addition, individualized support from educators of home-families enhanced this process, linking participants to facilitate social support over the ten weekly periods.

c. Skill Building- Skill building exercises were explored to improve participants' personal formation in managing daily problems and anxiety associated with their disability. Techniques included: identifying self-talk and belief systems, negative thought stopping, increasing opportunities for rewarding cognitive experiences such as effective problem solving (e.g., coping and self-reinforcing statements), learning experience how to avoid personal problems and interrupt them in the early stages of occurrence (e.g., through the use of specific movements or mental activities).

d. Educational Information about identity and existence - Sessions included general information about identity and existence, medication, identifying existing triggers such as meaning in life, and the opportunity to share experiences.

e) Physic and Mental Exercises - In each session is included one kind of exercise as Instant relaxation, the peacemaker prophetic, cerebral gymnastics.(Lindenfield Gael,1999: 50, 56, 58) Each of these exercises was aimed at relieving stress and tensionfrequently reported as precursors of mental or behavior problems/disturbances. The techniques used are considered to have lessened tension during the day, improved feelings of control, and reduced fear, indicating an overall greater sense of wellbeing.

Sessions were characterized by being: (a) inexpensive, (b) easily supported and complimentary to other rehabilitation programs, and (c) used terminology that was shared and understood. Each of these approaches imply that the 
responsibility is on the facilitator and participant to take an active role and responsibility in the program, sharing information, using role reversal, and developing dialog and strategies for openness. A folder containing each session's material, discussion questions and notepaper was brought to every session and supplemented by information/resources participants brought to share. The completed folders then formed each participant's own resource. On occasions; the group suggested the sessions be held in different locations of the city. This included parks in public spaces, which brought a more relaxed and informal atmosphere to the sessions. Informality is perceived as a non-threatening strategy which improves motivation and enhances openness. In this way, the sessions evolved to include opportunities for structured learning, as well as interacting in a more social context in natural environments. Educators from Home-Families were invited to provide assistance (e.g., managing problems).

7. Outcomes: Impact of neuro-psycho-education-existential group program

Data collection- Information on all participants was gathered from the following sources: through psychological records and observations, participant's reflections, use of home-works participants and reporting of assistants of program including to the educators themselves.

\subsection{Benefits of Participation in Weekly Intervention Program}

Psychological records and observations - it can be said that a considerable PPID have severe disabilities understanding and accepting the life inside the hard basket, so their existence means me and only me, this basket is mine, there is no space for others and then I don't want to stay anymore inside this basket, I want to go outside and then I want to turn back again to my basket and again I want to go out. PPID is a serious complication issue. Therefore, I bring the outcomes of the practical application.

-Findings indicate that "being" inside the so called "too strong basket", a lack of understanding and support are key areas of concern that place many people with PPID at risk of poor existence, continued mental crises, and reduced opportunities in rehabilitation and community reintegration.

-Developing personal, educational skills alongside effective daily management of PPID requires careful nurturing of participants allied to sensitivity to their needs especially not feeling that they are not just being but existing.

-This in turn have built a self-awareness and confidence such that they can manage their disordered behaviors by having a stimulus in programmed 
activities, the process of personal formation is enhanced mentioning here the help by giving a meaning in their life.

-Through weekly discussions, participants appeared to adjust the way they interacted with others inside the big group, but being divided in 6 mini-groups by five persons each, developing within contacts, and spoke with more freedom and confidence about their meaning in life.

-The program provided; a sense of purpose and direction for participants, opportunities to assist others, and provided a valued role in the group which is described as someone who actively listens, can provide suggestions and helpful strategies for solving problems. Supporting others, in addition to receiving support, is an important issue highlighted by this project pilot. For egg, one participant's desire was to meet others who have a family. For her, the chance to 'connect with others was keenly expressed because is a cornerstone for her future existence.

-Without a positive focus, the participants tended to lose direction and fall back in to periods of passivity, isolation, and disorder behavior.(especially with psychiatric disabilities).Facilitating greater openness to affective and cognitive stimuli required strategies to help manage this newfound awareness.

Participant's reflections- by the end of each daily activity, they were mostly able to express that they felt good or bad trying to give an answer about what they have understood or what they have not liked to other attitudes, behavior ecc and what can be done in the next activity. This because most of them have continually waited the participation in weekly program under the influence of a "good stress" in the sense that "will this program go on every Sunday" ..."etc....and then what after, will activity go on when the good weather be because we can not go out when is raining etc. Once the activity had begun, they said I have done the homework or one telling the reason of not doing it.

Reporting of educators - they have discussed and given further information on their minimal or moderate progress, confirming data from the impact of group program especially regarding doing home-works to which have shown a great distress to complete them or to be in time of parting which indicates that higher awareness of difficulties following in their routine life is often accompanied by emotional distress. It can be mentioned three cases when the younger couldn't come because of being ill or were preparing to their birthday feast, they have asked to the educators to write a letter of permission. They have reported that this kind of program has helped them mostly to manage better their care to them and have called it facilitating of their daily life care.

Reporting of three assistants with PPID- they have expressed good feelings toward being assistant of such an activity which has helped them to be centered but also to be the good model for the others, giving them hope that they can really live although their disability, they can make good things and they can manage 
better their life by having a positive stimulation, orientation, and being included in a social support

7.2. Factors which influenced the success of the intervention- The following features were observed to influence participant engagement in, and benefits gained through the intervention program:

(a) Safe environment- the setting is the most important part of group program, going out the basket into public spaces. From the psychological point of view there seems to be five levels that a clinician can work with most often. (1) The basic level is your environment, your external constraints. (2) You operate on that environment through your behavior. (3) Your behavior is guided by your mental maps and your strategies, which define your capabilities: (4) these capabilities are organized by belief systems - and (5) beliefs are organized by identity (Dilts R.1996). By interacting in helpful ways being in public spaces, participants could be stimulated and developed considerable strategies to deal with complex issues.

(b) Structure in sessions and materials- Holding sessions at set times, in a set location, with a set pattern and prepared materials provided a level of structure to which the participants responded well. Participants knew 'what to expect next, and could anticipate the events. Any change to this routine was kept to a minimum.

(d) Opportunity to communicate change, verbally and in writing- During sessions, participants had the opportunity to share personal experiences by talking with the group, being a valued group member whose contributions are valued and writing down (some representatives inside the group) events/feelings/behaviors in their worksheet. At times, this included examples of successful interactions with others with whom they had negative experiences in the past, and the desire talking to the strangers. Other examples include sharing how they were able to 'interrupt a bad action ' by changing their behavior.

\subsection{Barriers to Successful Involvement in Sessions of Group Program-} Observations revealed a number of issues that limited successful involvement in the program:

(a) Fatigue- A number of statements by participants illustrated the effects and causes of fatigue."I'm tired - I don't feel alert because of the drugs.", "I get tired very easily because I can't walk". Feeling 'tired', restricts ability to attend to stimuli, respond appropriately, and interact with others in a positively rewarding way. Intervention sessions are kept short, with breaks and chances to rest during activity.

(b) Transport-Isolation due to lack of transport was a common problem for some of participants. 
(d) Atypical behaviors- Feelings of being worried were reported due to, independence and roles, reliance on long term medications, inability to find and sustain meaningful employment. The following statement illustrate these feelings:"I get worried when I can't hear conversations."

Adjustment to disability- PPID may become increasingly anxious or depressed as a result of the threat and restrictions posed on their lives because of the disability. This process can be aided through this group program in the context of a model developed in public spaces. However, observing the impact and role of such a program demonstrates that interventions need to be continually sustained. The ten weekly group program sessions revealed an improvement in self-awareness of existing by participants, not simply being. They could say $\boldsymbol{I}$ and we are but also we can exist if we have a stimulus. At the end of the activity is developed at Village of Peace a cocktail cooked by them and educators and as a stimulus to their contributing in the activity is selected by themselves the Girl and the Boy of the Activity Road of Hope. However, as demonstrated by the results, these improvements are not sustained unless the intervention is maintained. Evidence show that there is no ideal program of intervention provision that redresses the complex mental health needs of PPID who live within community settings; personal formation is an ongoing activity. Group support must be ongoing, and provide a variety of different forms that are appropriate to the PPID. As time passes, these needs change and must be reflected in the nature of the support provided. Examples include changes in daily activities, change in mood state (egg passivity), changes in support structure (egg loss of someone support or development of new relationships) and continuous appropriate stimulus.

\section{Conclusions and Implications}

Evaluation of this project pilot indicates the following limitations:

- The sample size in this study was chosen to allow for group work, qualitative data collection and analysis and would need to be not more than 20 participants

- The 10 weekly program periods was selected to facilitate ongoing weekly participation, and accommodate the time constraints of a further project. In any future, further detailed results would be possible in a study of longer duration.

- A range of different types of PPID, including etiology of disability, type and symptomatic frequency were gathered from the 28 participants and this provides a greater range of variables, and potentially reveals important issues regarding the impact of this group program. 
- Small studies of this type create the potential for bias. Thus, the project pilot findings should be interpreted with this caveat in mind.

- Difficulties in communication, memory and self-awareness have the potential to limit the ability of the participant with PPID to accurately identify, report activity and its effects. In this population, communicating details regarding their stimulation type and frequency represents a significant problem.

- $\quad$ This project pilot has identified cases where symptomatic may have been 'missed' due to memory difficulties (inability to remember events due to disability or confusion), difficulty describing the meaning of the activity (again due to communication difficulties associated with the type of disability), and inability to identify or link an event or behavior with activity (e.g., recognize simple partial).

- The question must be raised as to whether the results from the intervention group are due to the group program effect. In order to establish this in future projects, multiple testing prior to intervention would ensure that selfreporting was stable and accurate prior to treatment. However, asking people with PPID to complete questionnaires to ensure reliable data is often considered impractical.

- Was the intervention group just responding to the increased personal formation? The need for further evaluation and design research is considered for a better implementation into practice. Future practices could attempt to include educators and other assistants in pre-training as a better introduction to the techniques and then examine the impact of NPEEGP for this group that has been historically and geographically isolated and excluded.

Acknowledgment: a special and heartily thank to all the participants and staff of Progetto Speranza who made possible the application of this professional practice by wishing to go on with the Road of Hope in their future life.

\section{References:}

-Dilts Robert (1996) So called Logjical Levels and Systemic NPL, retrieved from http://www.cnlpa.de/fileadmin/Fachartikel/1999-06_So-called-Logical-

Levels_Woodsmall.pdf

-Lindenfield Gael (1999) Emotional Confidence. Edited by Ingraf s.r.l,Piero GribaudiMilano (pg 50,56, 58)

-Ndoja Suela, 2012. A further advance in clinical perspective: An introduction to Neuro-PsychoEducation Model, Journal of Educational and Social Research, Special Issue, 
Copyright of Mediterranean Center of Social and Educational Research, Gruppo Atena.net Srl Edition, (pg 68)

-Nietzel T. Michael, Douglas A Bernstein, Richard Milich, Prentice Hall,(1991, 1987). Introduction to clinical psychology Third edition, A division of Simon and Schuster Englewood Cliffs, New Jersey 07632, (pg (374)

-Marzuoli Ugo, (1961).Psiche e condizionamento, Copyrights by Giangiacomo Feltrinelli Editor. Milano ( $g$ g 230-231) 